\title{
EVOLUÇÃO DA URBANIZAÇÃO EM BELO HORIZONTE (1918-2000) E RELAÇÕES ESPACIAIS COM OS INDICADORES SÓCIODEMOGRÁFICOS
}

\author{
Diego Rodrigues Macedo ${ }^{1}$ \\ Glauco Umbelino ${ }^{2}$
}

\section{Resumo}

Este artigo descreve os resultados da análise da correlação espacial entre a evolução da mancha de urbanização em Belo Horizonte e os índices sócio-demográficos. O objetivo principal é identificar em qual fase histórica da evolução da cidade atual localização dos moradores está associada, e correlacioná-la com índices que diferenciam a estrutura etária e a renda dos habitantes. Os dados sociodemográficos foram extraídos do Censo demográfico (2000), sendo mapeados em nível de setores censitários. As informações para expansão urbana foram extraídas de fotografias aéreas realizadas em cinco momentos anteriores ao Censo 2000. Essas informações foram interpretadas manualmente pela Secretaria de Planejamento Urbano da Prefeitura de Belo Horizonte através de um SIG, gerando um mapa com a evolução urbana. A Análise de Componentes Principais (ACP) foi utilizada na formulação dos índices de estrutura etária e renda. Foi utilizada a correlação de Pearson na interpretação os índices extraídos da ACP e as fases de crescimento urbano de Belo Horizonte. Os resultados mostram que as áreas mais antigas possuem uma população mais idosa. Também foi detectado que a renda não tem correlação com a evolução do crescimento urbano. Estes resultados mostram que no geral, os moradores envelhecem com seus domicílios, e desta maneira, a valorização das áreas mais consolidadas não necessariamente indica a presença de uma população de maior renda.

Palavras-chave: SIG, estatística espacial, análise de componentes principais.

\footnotetext{
${ }^{1}$ Universidade Federal de Minas Gerais - Instituto de Geociências - Laboratório de Estudos Territoriais. Av. Antônio Carlos, 6.627 - 31270-901 - Belo Horizonte - MG (diegorm@ufmg.br)

${ }^{2}$ Fundação João Pinheiro - Centro de Pesquisas Aplicadas. Alameda das Acácias, 70 - 31275-971 - Belo Horizonte MG (glauco@fjp.mg.gov.br)
}

Cadernos do Leste 


\section{1- INTRODUÇÃO}

A utilização de Sistemas Informativos Geográficos (SIG's) integrando dados sóciodemográficos a informações advindas de sensores remotos causaram um impacto significativo nos estudos da dinâmica urbana nos últimos anos. Suas ferramentas têm propiciado aos pesquisadores um ganho expressivo de informações, permitindo uma melhor compreensão da ocupação populacional em diversas regiões, com destaque às áreas urbanas. Em estudos de planejamento urbano, os SIG's têm diversas utilidades, desde trabalhos na escala nacional, até a escala intra-urbana, a começar pelo georreferenciamento de informações cadastrais de equipamentos públicos (educação e saúde) e de mortalidade, passando pela elaboração de surveys e índices, até estudos relacionados à dinâmica evolutiva de áreas urbanas (Davis e Fonseca, 2001; Souza e Torres, 2003).

O aprimoramento tecnológico das ferramentas de geoprocessamento permitiu a integração de dados espaciais de diversas fontes, possibilitando realizar análises cada vez mais complexas. Atualmente, é possível espacializar diversas informações e testar estatisticamente as relações entre as variáveis espaciais, desde que exista um identificador único (como municípios, setores censitários, endereços, etc.). No Brasil, a menor unidade de análise de dados sóciodemográficos que possuam abrangência nacional, confiabilidade e periodicidade são os setores censitários do IBGE. Desta maneira, a integração destas informações pode elucidar o comportamento espacial de variáveis demográficas nas metrópoles brasileiras, como em Belo Horizonte (figura 1), que é o foco deste estudo.

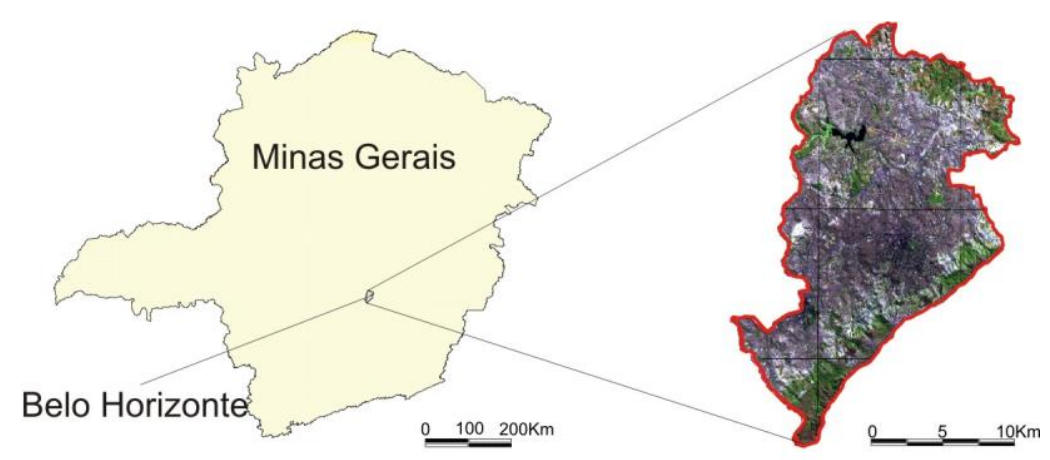

Figura 1. Localização do município de Belo Horizonte.

Neste contexto, o objetivo deste artigo é identificar em qual fase da evolução da mancha urbana de Belo Horizonte cada setor censitário pertence, e correlacionar sua localização com o perfil sócio-demográfico de seus moradores. Serão utilizados os dados presentes no resultado do universo do censo demográfico de 2000 (IBGE, 2002) integrados com a malha de setores censitários, que permitirão a geração de índices sócio-demográficos, além do mapeamento das Cadernos do Leste 
manchas urbanas de Belo Horizonte elaboradas através da interpretação manual de imagens aerorestituídas pretéritas. Serão testadas duas hipótese levantadas por Ferreira (2004):

-Hipótese (1): O perfil etário dos residentes e chefes de domicílios acompanha o desenvolvimento da cidade, ou seja, as áreas mais antigas possuem maior proporção de moradores idosos, sendo que estes também são os responsáveis pelos domicílios. Também há menor proporção de jovens e filhos, pois estes já constituíram novas famílias, ou;

- Hipótese (2): O aumento da riqueza familiar está relacionada ao ciclo de vida dos domicílios, e neste caso, os domicílios mais antigos teriam melhores condições econômicas. Além disto, pode-se inferir que as áreas mais antigas estão consolidadas, possuindo uma melhor infraestrutura urbana, ocasionando uma valorização dentro do espaço urbano, e conseqüentemente, atraindo moradores com melhores condições econômicas.

Sobre o município analisado, vale ressaltar que Belo Horizonte foi a primeira capital planejada do país. Esta foi construída para desvincular a idéia da capital de Minas Gerais de uma cidade imperial (Ouro Preto). Desde o projeto inicial, visava-se atrair para dentro dos limites da área urbana funcionários públicos e membros da elite. Os lotes não eram acessíveis as famílias mais humildes, formadas por operários e pelos antigos habitantes do Arraial de Belo Horizonte (Curral Del Rey). Desta maneira, o mercado controlou a expansão urbana da capital, fazendo com que esta fosse realizada da periferia em direção ao centro (Costa, 1994; Monte-Mór, 1994).

Dentro do complexo processo de evolução urbana de Belo Horizonte, pode-se citar alguns momentos históricos cuja conjuntura moldou seu tecido urbano. Até a década de 1920, a ocupação era restrita a algumas áreas na região central. $O$ alto preço dos lotes impedia a população de baixa renda de ocupar a zona urbana, e estas ocupavam as áreas periféricas ao norte. Na década de 1930 ocorre a criação do pólo industrial mineiro, a Cidade Industrial, determinando um eixo de expansão urbana no sentido oeste em relação ao centro de Belo Horizonte. Desde modo ocorre um crescimento urbano intenso até a década de 1950, principalmente além dos limites da Avenida do Contorno. Logo a cidade se expande em direção a dois eixos preferenciais: o eixo oeste, industrial, e o eixo norte de habitações de alta categoria (Pampulha) (Monte-Mór, 1994). Na década de 1970 as mudanças na dinâmica econômica e espacial brasileira também atingem Belo Horizonte, fazendo com que a população crescesse de 693.328 habitantes na década de 1960 para 1.255.415 habitantes na década seguinte (Costa, 1994). Parte deste crescimento se deu através de fluxos migratórios, majoritariamente formados por uma população de baixa renda, que tendeu a fixar moradia em bairros (geralmente loteamentos populares) distantes da área central (Silva et al., 2008). Nas décadas seguintes houve a expansão 
das áreas periféricas, e atualmente, o sítio urbano não possuí mais áreas de expansão urbana. As grandes áreas ainda não urbanizadas são parques e áreas de proteção, possuindo o status non adificandi, de acordo com a Lei de Parcelamento, Uso e Ocupação do Solo de Belo Horizonte (Belo Horizonte, 2000).

\section{2- METODOLOGIA}

A primeira etapa de trabalho foi identificar as manchas de ocupação urbana do município de Belo Horizonte em momentos históricos. Estas foram mapeadas pela Secretaria de Planejamento Urbano de Belo Horizonte a partir de fotografias aéreas provenientes de levantamentos realizados durante o século passado, em momentos chave no processo de ocupação do sítio: 1918, 1936, 1950, 1977 e 1995. Estas imagens foram interpretadas manualmente e posteriormente vetorizadas, resultando em um mapa com a evolução das manchas urbanas de Belo Horizonte (PBH, 2000). Estes dados foram integrados a malha de setores censitários, ou seja, cada setor teve adicionado ao seu banco de dados o ano no qual sua ocupação foi observada pela primeira vez. Como o censo foi coletado em 2000, os setores censitários que não foram ocupados em nenhum momento pretérito foram associados à data do ultimo censo, com a exceção aos que não possuíam domicílios, sendo estes eliminados da análise.

A segunda etapa foi extrair dos dados censitários um índice que representasse a estrutura etária dos domicílios e outro que melhor refletisse as condições de renda. Utilizou-se a técnica de análise estatística de componentes principais (ACP) para se extrair estes índices.

O objetivo principal da ACP é a obtenção de um pequeno número de combinações lineares (componentes principais) de um conjunto de variáveis, que retenham o máximo possível da informação contida nas variáveis originais. Os componentes são extraídos na ordem do mais explicativo para o menos explicativo. Teoricamente, o número de componentes é sempre igual ao número de variáveis. Entretanto, alguns poucos componentes são responsáveis por grande parte da explicação total. Uma vez determinado os componentes principais, seus valores numéricos (scores) podem ser calculados para cada elemento amostral. Deste modo, pode-se posteriormente utilizar na análise dos dados técnicas estatísticas usuais, como análise de variância ou análise de regressão, como sugerido por Mingoti (2005). 
Como as variáveis utilizadas não possuem a mesma escala, foi necessário padronizar os doados através do método Z-Score, conforme a equação 1:

$$
z=\frac{x-x_{m}}{\sigma}
$$

Nesta equação, Z é o valor padronizado da variável, X é valor inicialmente encontrado da variável para cada setor, Xm é o valor médio da variável (média dos valores obtidos para todos os setores) e $\sigma$ é o desvio padrão da variável na amostra.

Os indicadores utilizados para construir o índice de estrutura etária dos setores censitários se encontram na tabela 1:

Tabela 1. Indicadores do índice de estrutura etária

\begin{tabular}{lcc}
\hline \multicolumn{1}{c}{ Indicador } & \multicolumn{2}{c}{ Variável } \\
\cline { 2 - 3 } Razão de jovens & $\begin{array}{c}\text { Número de moradores com } \\
\text { idade entre 0-19 anos }\end{array}$ & Denominador de moradores \\
\hline Razão de idosos & $\begin{array}{c}\text { Número de moradores com } \\
\text { idade acima de 60 anos }\end{array}$ & Total de moradores \\
\hline Razão de chefe de domicílio & $\begin{array}{c}\text { Número de chefes de domicílios } \\
\text { com idade entre 15-60 anos }\end{array}$ & Total de domicílios \\
adulto & $\begin{array}{c}\text { Número de chefes de domicílios } \\
\text { com idade acima de 60 anos }\end{array}$ & Total de moradores \\
$\begin{array}{l}\text { Razão de dependencia em ao chefe de domicílio } \\
\text { idoso }\end{array}$ & Número de filhos residentes & Total de moradores \\
\hline Razão de filhos & &
\end{tabular}

Fonte: IBGE, 2002.

Destaca-se que o indicador construído pela razão entre moradores adultos (idade entre 20-59 anos) e o total de moradores foi excluído da análise, por ser um dado complementar ao indicador de razão de jovens e de razão de idosos, como recomendado por Mingoti (2005). O indicador de razão de chefe de domicílio idoso (acima de 60 anos) pelo total de domicílios é complementar ao indicador de razão de chefe de domicílio adulto, e pelo mesmo motivo, foi excluído da análise.

Os indicadores disponíveis no censo para avaliar a condição econômica da população para a escala deste trabalho são bem restritos. As únicas variáveis que podem ser utilizadas para este objetivo são a renda e a escolaridade do chefe do domicílio (IBGE, 2002). Neste caso, criaram-se três indicadores, expostos na tabela 2: 
Tabela 2. Indicadores do índice de renda

\begin{tabular}{lcc}
\hline \multicolumn{1}{c}{ Indicador } & \multicolumn{2}{c}{ Variável } \\
\cline { 2 - 3 } $\begin{array}{l}\text { Média dos anos de estudo do } \\
\text { chefe do domicílio }\end{array}$ & $\begin{array}{c}\text { Total de anos estudados pelos } \\
\text { chefes de domicílios }\end{array}$ & Total de domicílios \\
\hline $\begin{array}{l}\text { Rendimento médio do chefe do } \\
\text { domicílio }\end{array}$ & $\begin{array}{c}\text { Renda total dos chefes de } \\
\text { domicílios }\end{array}$ & Total de domicílios \\
\hline $\begin{array}{l}\text { Rendimento do chefe do } \\
\text { domicílio per capta }\end{array}$ & $\begin{array}{c}\text { Renda total dos chefes de } \\
\text { domicílios }\end{array}$ & Total de moradores \\
\hline
\end{tabular}

Fonte: IBGE, 2002.

Os índices calculados foram integrados a malha de setores censitários através de ferramentas de geoprocessamento, ou seja, para cada setor tem-se um momento vinculado e dois índices. Desta maneira é possível realizar a análise estatística de correlação espacial através de pacotes estatísticos convencionais. Neste caso, foram determinados os coeficientes de correlação de Pearson (r) entre os índices criados e os estágios evolutivos de Belo Horizonte.

\section{3- RESULTADOS E DISCUSSÃO}

Os resultados da análise de principais componentes para o índice etário indicam que a componente C1 explica cerca de $80 \%$ da variância dos dados, e desta maneira a redução das variáveis é satisfatória (Mingoti, 2005). A tabela 3 mostra os resultados dos coeficientes dos indicadores para a componente principal C1:

Tabela 3. Coeficientes para o componente $\mathrm{C} 1$ do índice de estrutura etária

\begin{tabular}{lr}
\hline Indicador & Coeficiente \\
\hline Razão de jovens & 0,896918 \\
Razão de idosos & $-0,948213$ \\
Razão de chefe de domicílio adulto & 0,897820 \\
Razão de dependencia em relação ao chefe de domicílio idoso & $-0,963597$ \\
Razão de filhos & 0,774549 \\
\hline
\end{tabular}

Os elevados valores dos coeficientes (acima de 0,7, independente do sinal) permitem afirmar que a C1 pode explicar o comportamento de todas as variáveis analisadas. Entretanto, os valores negativos para os indicadores razão de idosos e razão de dependência em relação ao chefe de domicílio idoso, indicam que os setores que possuem o índice etário negativo apresentam maior proporção de idosos em sua estrutura, além de possuir muitos moradores dependentes destes idosos. Os valores positivos indicam a maior presença de jovens, filhos e adultos chefiando os domicílios. Valores próximos a zero mostram um equilíbrio entre as duas situações. Neste caso, o índice construído é válido para testar a Hipótese (1) formulada. 
Em relação ao índice de renda, o valor da variância explicada pela C1 (93\%) também é suficiente para definir seus coeficientes como os valores ponderados para a construção do índice. A tabela 4 mostra os coeficientes para a C1:

Tabela 4. Coeficientes para o componente $\mathrm{C} 1$ do índice de renda

\begin{tabular}{lr}
\hline Indicador & Coeficiente \\
\hline Média do anos de estudo do chefe do domicílio & $-0,939314$ \\
Rendimento médio do chefe do domicílio & $-0,977343$ \\
Rendimento do chefe do domicílio per capta & $-0,984658$ \\
\hline
\end{tabular}

Os resultados dos coeficientes da C1 mostram que quanto menor o índice, melhor as condições de renda dos moradores, sendo, portanto um indicativo inversamente proporcional à condição econômica dos domicílios. Neste caso, este índice pode ser utilizado para testar a Hipótese (2) deste artigo.

Em relação ao resultado da correlação de Pearson entre os estágios de evolução da mancha urbana e o índice etário, a figura 2 ilustra a distribuição dos valores em cada ano:

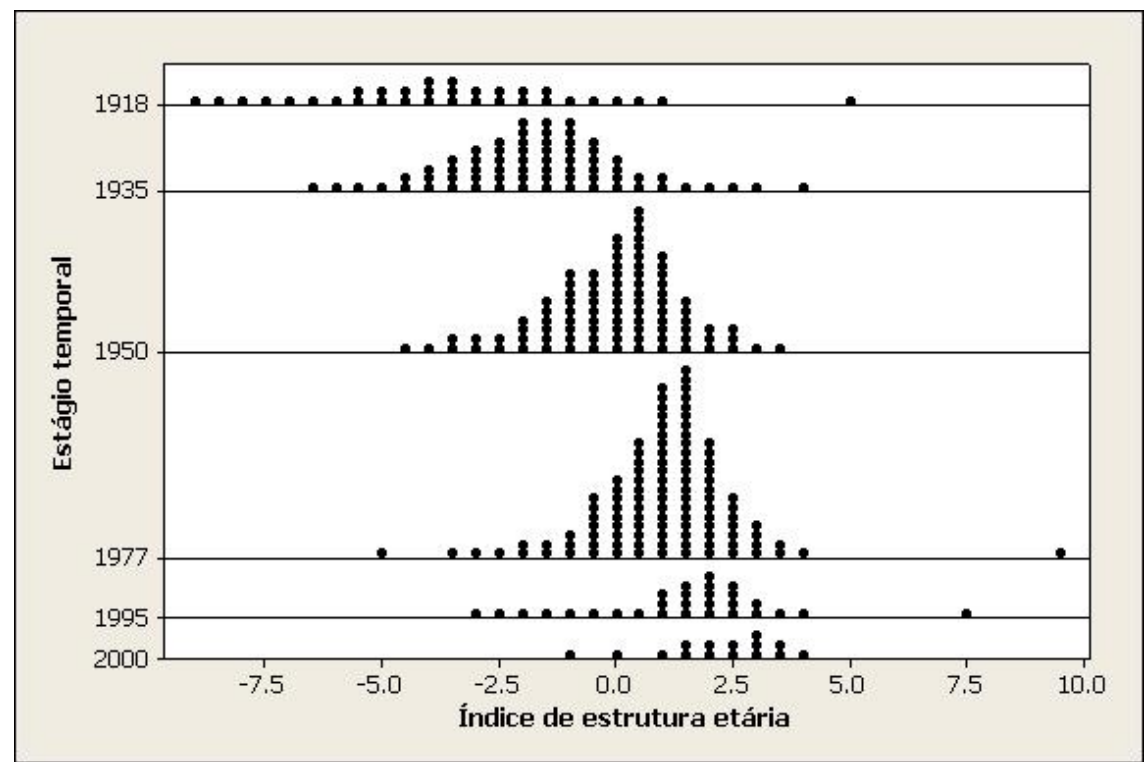

Figura 2. Dotplot da distribuição dos valores do índice etário nos anos estudados.

Nota-se na figura acima que a cada ano a maior freqüência das observações ocorre em valores do índice sucessivamente maiores, indicando que nas áreas mais antigas é maior a proporção dos idosos e dos domićlios chefiados por idosos. Estes valores possuem valores positivos em áreas cuja ocupação é mais recente, observando uma inversão da estrutura nos setores ocupados a partir da década de 1970. O teste de correlação confirma o resultado do gráfico, pois o r observado é de 0,7 , ou seja, existe uma alta correlação entre os estágios temporais e a estrutura etária. 
A figura 3 ilustra a distribuição dos valores do índice de renda pelos estágios temporais:

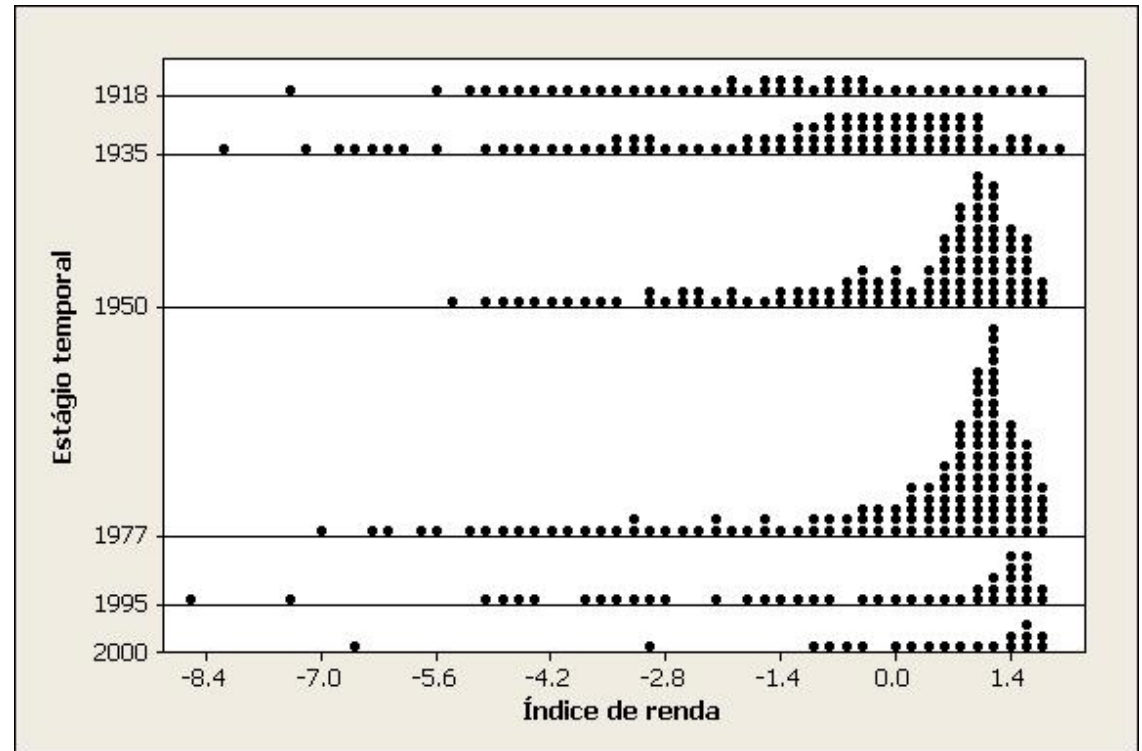

Figura 3. Dotplot da distribuição dos valores do índice de renda nos anos estudados.

Em relação à renda, o gráfico apresenta uma distribuição ampla dos valores do índice nos estágios temporais observados. Neste caso, os valores não correspondem aos períodos temporais, pois se observa muitos setores com índices altos e baixos em todos os anos. A análise estatística também mostra uma correlação baixa entre os estágios e o índice de renda $(r=0,33)$.

Desta maneira, a Hipótese (1), na qual as áreas cujos setores mais antigos possuem maior proporção de idosos e chefes idosos, e menor proporção de adultos responsáveis por domicílios, jovens e filhos é mais coerente.

A figura 4 auxilia na interpretação dos resultados, corroborando a análise estatística apresentada. Na figura 4A, o índice de renda foi espacializado indicando na legenda a divisão dos setores em duas classes: acima e abaixo de zero. Deve-se ressaltar que o índice tem valor inverso à condição real de renda. Comparando com a figura 4B, não é perceptível qualquer relação entre maior renda e a evolução na ocupação urbana dos setores. A figura 4C indica que as áreas com baixo índice etário (abaixo de zero, ou seja, com maior proporção de idosos e chefes de domicílios idosos) estão visivelmente relacionadas com as três primeiras fases da expansão da mancha urbana de Belo Horizonte, conforme os resultados da análise estatística. 


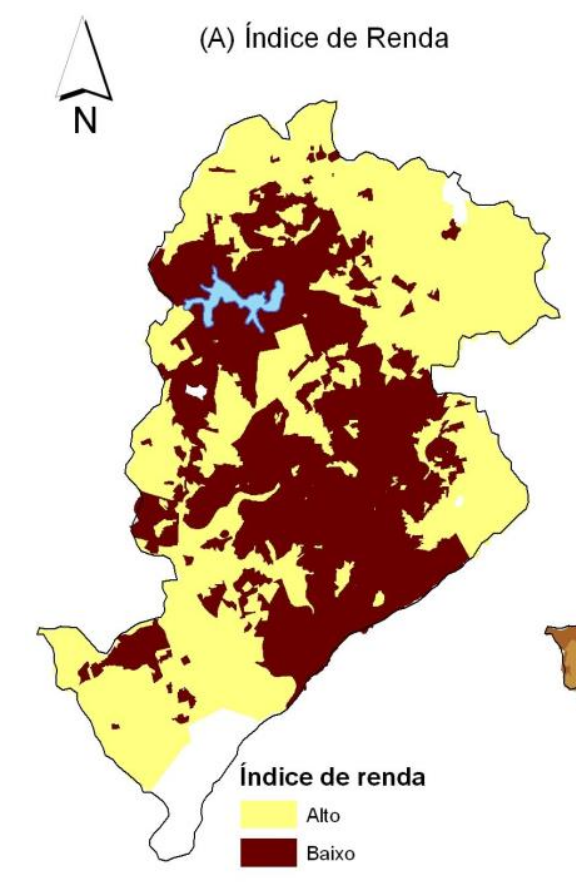

(B) Evolução da Mancha Urbana

(C) Índice de Estrutura Etária
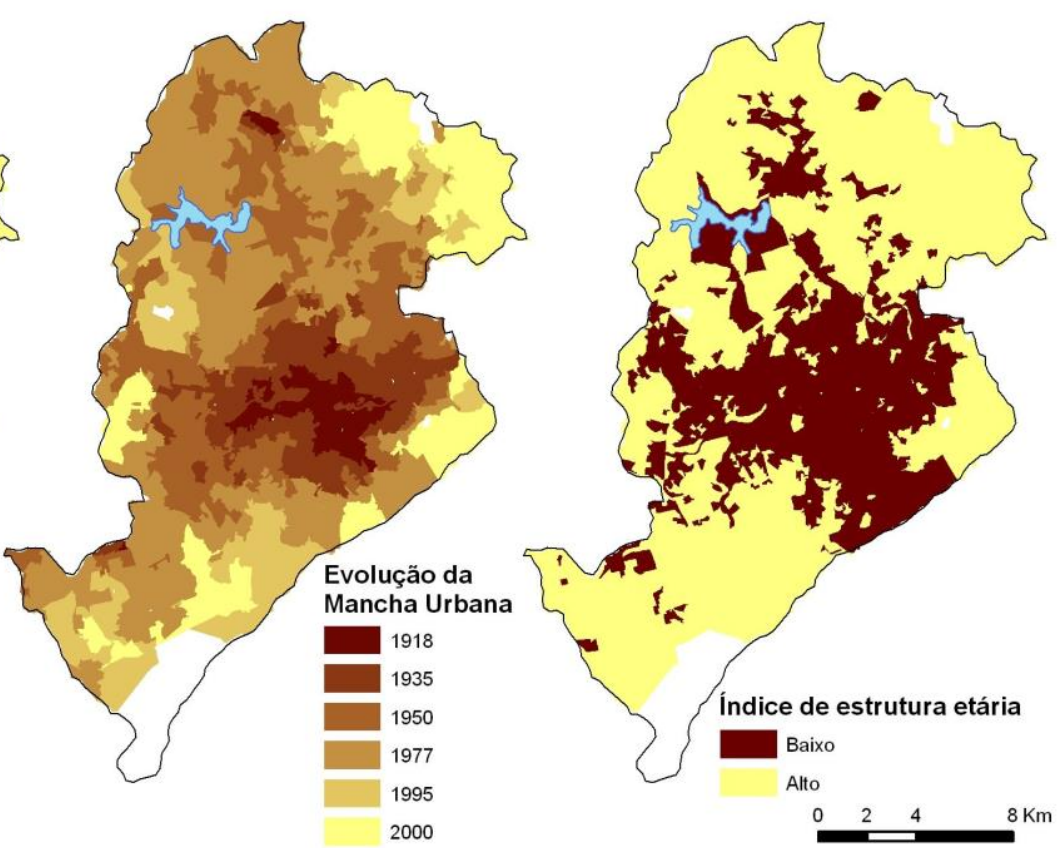

Figura 4. Espacialização dos resultados encontrados.

Portanto, são coerentes as considerações aventadas por Ferreira (2004), nas quais as famílias envelhecem em seus domicílios. Deste modo, após os moradores se fixarem em suas residências e constituírem sua unidade familiar, a tendência é que não migrem, permanecendo até o final de seu ciclo de vida. Como a expectativa de vida nas regiões metropolitanas brasileiras vem aumentando, a taxa de desocupação destes imóveis vem diminuindo e acredita-se que essa tendência será mantida no curto prazo.

A hipótese de que as regiões mais antigas de Belo Horizonte (mais próximas da região central e mais bem dotadas de infra-estrutura urbana) atraiam uma população com renda superior não foi observada através da análise dos dados. Deve-se ressaltar que áreas nobres localizadas na zona sul e na região da Pampulha só foram ocupadas a partir da década de 1950, além do recente eixo-sul, o que em parte justifica a não-correlação entre renda e estágios da ocupação urbana.

\section{4- CONCLUSÕES}

O estudo realizado cumpriu o objetivo de elucidar o fenômeno intra-urbano analisado. A integração dos pacotes de estatística tradicional com as ferramentas de geoprocessamento e sensoriamento remoto permitem um ganho expressivo nestes estudos.

Este artigo teve que se limitar a disponibilidade das imagens aéreas (uma periodicidade maior de imagens poderia contribuir para a geração de resultados mais robustos), além da Cadernos do Leste 
limitação dos dados censitários em 2000, pois o IBGE não disponibiliza os microdados do questionário da amostra para os setores censitários. De qualquer forma, o método permitiu uma melhor compreensão da ocupação urbana do município analisado e pode ser aplicado e adaptado para demais áreas urbanizadas que possuam fotografias aéreas de vários períodos.

\section{REFERÊNCIAS BIBLIOGRÁFICAS}

Belo Horizonte. Lei $\mathrm{n}^{\circ}$ 8.137, de 21 de dezembro de 2000. Consolida a lei $\mathrm{n}^{\circ}$ 7.166, que estabelece normas e condições para parcelamento, ocupação e uso do solo do município de Belo Horizonte. In: Diário Oficial do Município de Belo Horizonte, Belo Horizonte, 2000.

Costa, Heloísa S. M. Habitação e Produção do Espaço em Belo Horizonte. In: Monte-Mór (coord.) Belo Horizonte: Espaços e Tempos em Construção. Belo Horizonte: Cedeplar/Pbh, 1994, p.51-77.

Davis, Clodoveu, Fonseca, Frederico. Introdução aos Sistemas de Informação Geográficos. Belo Horizonte: Departamento de Cartografia/UFMG, 2001.

Ferreira, Frederico P. M. Evolução Urbana e Demográfica do Envelhecimento em Belo Horizonte. Revista Brasileira de Estudos Urbanos e Regionais, v. 4, p. 71-82, 2004.

Instituto Brasileiro de Geografia e Estatística - IBGE. Censo Demográfico de 2000 Agregado por Setores Censitários dos Resultados do Universo. Rio de Janeiro: IBGE, 2002.

Mingoti, Sueli. Análise de dados através de métodos de estatística multivariada: uma abordagem aplicada. Belo Horizonte: Editora UFMG, 2005. 297 p.

Monte-Mór, Roberto. Belo Horizonte: A Cidade Planejada e a Metrópole em Construção. In: Monte-Mór, Roberto (coord.) Belo Horizonte: Espaços e Tempos em Construção. Belo Horizonte: Cedeplar/Pbh, 1994, p. 11-28.

Prefeitura de Belo Horizonte - PBH. Mapa 4.1 - Evolução da Ocupação e da Mancha Urbana 1918/35/50/77/95. Belo Horizonte, 2000. Escala 1:300.000. Disponível em http://portalpbh.pbh.gov.br/pbh/ecp/comunidade.do?evento=portlet\&pIdPlc $=$ ecpTaxonomia MenuPortal\&app=estatisticas\&tax $=9091 \&$ lang $=p t \_B R \& p g=5922 \& \operatorname{taxp}=0 \& i d$ Conteudo $=17982$ $\& c h P l c=17982$. Acesso em: 16 de julho de 2008.

Silva, Harley; Amaral, Pedro V. M.; Simões, Rodrigo F. Vários Horizontes: Infra-estrutura, Habitação e Regionalização na Capital Mineira. In: Seminário sobre a Economia Mineira, XIII, 2008, Diamantina. Anais... Belo Horizonte: Cedeplar/UFMG, 2008.

Souza, Gustavo; Torres, Haroldo. O estudo da metrópole e o uso de informações georreferenciadas. São Paulo em Perspectiva, v. 17, n. 3-4, 2003. Disponível em: $<$ http://www.scielo.br/scielo.php? script $=$ sci_arttext\&pid=S0102-883920030003000 $05 \& \operatorname{lng}=$ en\&nrm $=$ iso $>$. Acesso em: 27 set. 2006. 